\title{
Image Reconstruction Based on Combination of Wavelet Decomposition, Inpainting and Texture Synthesis
}

\author{
Huawei CHEN \\ Tokyo Institute of Technology, \\ 2-12-1, O-okayama, Meguro-ku, Tokyo, Japan \\ chen_hua_wei@yahoo.com
}

\author{
Ichiro Hagiwara \\ Tokyo Institute of Technology, \\ 2-12-1, O-okayama, Meguro-ku, Tokyo, Japan
}

\begin{abstract}
Digital inpainting provides a means for reconstruction of damaged portions of an image. Although the inpainting basics are straightforward, most inpainting techniques published in the literature are only suitable for remarkable small portion or smooth color image. In order to avoid such shortcomings, we present a new algorithm for digital reconstruction based on combination of wavelet decomposition, Surface-based/PDE-based inpainting and texture synthesis. In which, wavelet transform at first decomposes the image into high frequency and low frequency level parts. Subsequently, CSRBF which is generally used for surface interpolation or PDE-based inpainting is employed for low frequency level and texture synthesis is used for high frequency level. It results in that not only slightly portion but also the common blotched image can be reconstructed with high quality. Especially, our algorithm makes many difficult cases for other methods possible.
\end{abstract}

\section{Keywords}

Inpainting, CSRBF, Wavelet transform, Texture Synthesis

\section{INTRODUCTION}

Digital photograph has been becoming popular vision device to produce electronic images as the alternative of traditional analog image. In addition to the immediate availability of photos for viewing and/or electronic transfer to an editorial office, digital cameras surpass in various respects such as ease to store and copy without loss of quality for the next upcoming decades. Although these advantages may sound great, one has to consider that a large amount of analog images have to be digitized in order to remain for ever, as well antique artifact or calligraphic works needs to be scanned. Unfortunately, this material often exhibits defects such as scratches or blotches. Even with respect to digital photograph or videos, there are generally many disturbing obstacles or artifacts to be removed, for instance, subtitles, logos, and physical objects

Permission to make digital or hard copies of all or part of this work for personal or classroom use is granted without fee provided that copies are not made or distributed for profit or commercial advantage and that copies bear this notice and the full citation on the first page. To copy otherwise, or republish, to post on servers or to redistribute to lists, requires prior specific permission and/or a fee.

Posters proceedings ISBN 80-86943-04-6

WSCG'2006, January 30-February 3, 2006

Plzen, Czech Republic.

Copyright UNION Agency - Science Press such as wires and microphones.

Image reconstruction literatures presented until now can be categorized into two approaches, PDEbased [Bertalmio 00] and Surface-based approach [Nikita 02]. Preserving isophotes is, however desirable in PDE-based approach, never perfectly attained in practice. The main problem is that both isophote estimation and information propagation are subject to numerical diffusion. In contrast to PDE, the surface-based approach is fast and simple to implement. However, this method has no provisions for preserving the isophotes' directions. Highgradient image areas generally are blurred or even not to converge. As another disadvantages, the inpainted region, in general, will be blur or smoothed in unnatural manner as the missed region is considerable large or image gradient is very sharp.

In order to avoid drawbacks mentioned above concerning with two methods, we proposed a new convenient method to automatically repair damaged areas in digitized photographs. The original photo is firstly decomposed into high-frequency and lowfrequency parts based on wavelet multi-resolution decomposition. Image inpainting, texture synthesis and noise removal are adopted for low-frequency and high-frequency respectively. The efficiency of proposed reconstruction method is to be clarified by diverse practical implementations. 


\section{Overview of Our Method}

As mentioned in the introduction, blemished photograph includes not only photograph with small spot or scratch which can be considered noise but also that with large damaged area. Image inpainting or reconstruction algorithm is necessary to take above two cases into consideration. Under such requirements, one novel and convenient algorithm combining the texture synthesis and inpainting is proposed as shown in Fig.1:

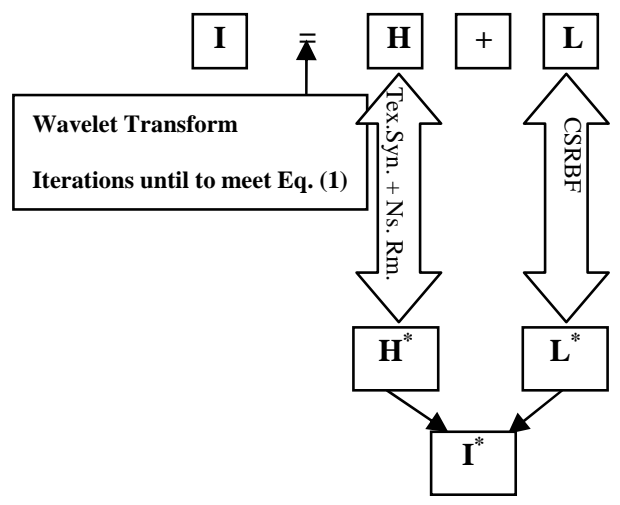

Fig.1 Procedure of proposed method (Tex. Syn.: Texture Synthesis; Ns. Rm.: Noise Removal)

1) The input image $I$ is decomposed into a high frequency part $H$ and low frequency part $L$ by wavelet multi-resolution decomposition.

2) In order to guarantee the property of the inpainted photograph and availability of inpainting method (surface-based or PDE-based), whether or not the gradient $G d_{k}$ of $k$ pixel to neighboring pixels exceeds the threshold is used to determine the iteration times of decomposition.

$$
G d_{k}=\frac{\sum\left|C_{i}-C_{k}\right| / 255}{N} \leq \text { threshold }
$$

where $C_{i}$ is color value of $i$ pixel; $N$ is the number of neighboring pixels. The threshold is approximately set to be 0.2 .

3) With respect to high frequency parts $H$, multiresolution texture synthesis is implemented to fill inside the masked areas.

4) With respect to low frequency part $L$ in general denoted $\boldsymbol{L} \boldsymbol{L}$, local CSRBF (Compactly Supported Radial Basis Function) developed on our own is used to modify the masked area.

5) Wavelet transform is frequently used to eliminate the random error in original signal. In the duration of image reconstruction, we also employ it to eliminate the small spot considered as noise.

6) Finally, the image $I^{*}$ will be reconstructed by use of inverse discrete wavelet transform.

\section{Experiment Result}

Fig. 2 shows the inpainting results for damaged Japanese cherry tree in which the boundary color of damaged area change more remarkably as compared with Lena photograph depicted above. The original Japanese cherry tree image in which the damaged area is approximately identical to Lena in size is shown in Fig. 2(a). Fig.2 (b) and (c) show the reconstructed results on basis of iterative gradient interpolation algorithm proposed by Bertalmio $\mathrm{M}$. and CSRBF respectively. Although they can be well adapted to above Lena image, both fail to reconstruct damaged Japanese cherry tree image. As the reason, it can be regarded as that the boundary color surrounding the damaged area changes significantly and the size of damaged area becomes broader. In contrast, the proposed combination reconstruction results in one desirable image which nearly exhibits damaged mark as shown in Fig.2(d). In addition, third-level wavelet transform is employed in the duration of decomposition. That is to say, the shaper boundary of damaged area leads to much more decomposition times in order to make the CSRBF available.

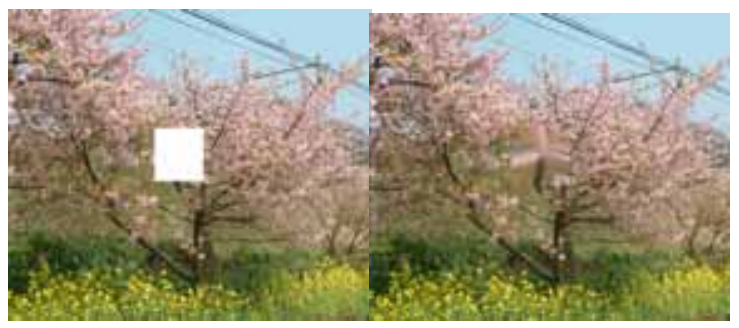

(a) Original Japanese cherry tree (b) Bertalmio M.

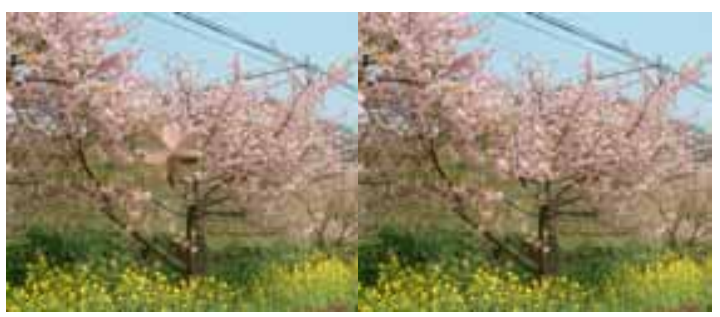

(c) $\mathrm{CSRBF}$

(d) Our Combination Approach

Fig. 2 Image Inpainting for Japanese Cherry Tree

\section{REFERENCES}

[Bertalmio 00] Bertalmio M., Sapiro G., Caselles V., Ballester C., Image Inpainting, SIGGRAPH 2000, p.417-424.

[Nikita 02] Kojekine N., Hagiwara I., and Savchenko V., Software Tools Using CSRBFs for Processing Scattered Data, Computer \& Graphics, pp. 309-317, 2002. 